\title{
Dynamiken im digitalen Wandel. Herausforderungen bezüglich Medien, sozialer Prozesse und Demokratie
}

\author{
Andrea Schaffar ${ }^{1,2,3}$ (D) \\ Online publiziert: 29. Juli 2019 \\ (c) Der/die Autor(en) 2019

\section{Zusammenfassung}

Digitalisierung und medialer Wandel sind gesellschaftliche Phänomene, die seit Längerem im öffentlichen Diskurs ihren fixen Platz einnehmen. Nicht nur veränderte der technologische Fortschritt zwischenmenschliche Kommunikation und deren Muster, auch alle weiteren Lebens- wie auch Arbeitsbereiche sind inzwischen digital durchwirkt. Reale, mediale und digitale Realitäten sind mittlerweile verschmolzen und können deshalb auch nicht mehr nur für sich genommen analysiert werden. Enorme Veränderungen ergaben und -geben sich dabei auch für Organisationen. Jedes Unternehmen, Organisation oder Institution existiert inzwischen auf einer reellen und physischen, wie auch digitalen und virtuellen Ebene. Insbesondere Medien und die dazugehörigen Medienunternehmen spüren diesen Druck. Als eine der Säulen, häufig als „,vierte Gewalt im Staat“ tituliert, obliegt ihnen eine wichtige demokratische Funktion: Die Kontrolle der Machthabenden und die Wahrung von Meinungs- und Pressefreiheit. Dieser Beitrag der Zeitschrift Gruppe. Interaktion. Organisation. diskutiert die Auswirkungen des digitalen und medialen Wandels, den dadurch bedingten Veränderungen in Gesellschaft und Organisationen und geht der Frage nach, welchen Beitrag die Gruppendynamik und ihre Settings dazu leisten kann.

Schlüsselwörter Digitalisierung $\cdot$ Medialer Wandel $\cdot$ Mediengeschichte $\cdot$ Soziale Dynamiken $\cdot$ Neue Lernformate

Mag.a Dr.in Andrea Schaffar

andrea.schaffar@univie.ac.at, andrea@projektbuero.at,

andrea@fiz.ac.at

1 Projektbüro XO KG, Seidengasse 28, 1070 Wien, Österreich

2 FiZ Forschungsinstitut Zivilgesellschaft, Seidengasse 28, 1070 Wien, Österreich

3 Institut für Publizistik und Kommunikationswissenschaft, Universität Wien, Währinger Straße 29, 1090 Wien,

Österreich 


\title{
Digital dynamics and change: Challenges concerning media, social processes and democracy
}

\begin{abstract}
Digitalization and change within the sphere of media have been societal phenomena in the midst of public discourse for years. The technological changes not only influence direct communication between people, but intervene in all other spheres of society, work and private life. One can't conceptualize real life and digital life apart. Both interact, intervene and are part of each other. Analyzing digitalization always means to rethink social processes and dynamics in real life as well in digital domains. People interact directly as well as digitally and organizations therefore also exist on these levels. Media and its organizations are especially challenged to cope with these requirements. As an important factor for democracy they have to watch over the freedom of press and opinions as well as being responsible to control and observe the ones who are in power. This contribution to the journal Gruppe. Interaktion. Organisation. discusses the outcome and terms of digital change and the consequences for the field of media and its organizations. An insight into media literacy and its connections to these discourses provides a perspective on similarities in the process to obtain media literacy as well as competences towards social dynamics. New designs for organizational labs with implemented digital elements display an opportunity to provide new ways in developing democratic culture and approaches within society with a practice based method.
\end{abstract}

Keywords Digitalization $\cdot$ Medial change $\cdot$ Media history $\cdot$ Social dynamics $\cdot$ New learning designs

\section{Demokratie- und Medienbildung im Lernsetting sozialer Prozesse}

Digitale und technologische Entwicklungen brachten die Notwendigkeit andersartiger Organisationsansätze und -strukturen mit sich. Zwischenmenschliche, wie auch mediale, Kommunikationswege wurden mit der Verbreitung von sozialen Medien grundlegend verändert und vormals bestehende Ordnungen auf den Kopf gestellt. Damit einhergehend veränderten sich einerseits die Organisationsprinzipien von Demokratien, anderseits aber jene von deren Kontrollelementen, den Medien. Das Lernsetting des Organisationslaboratoriums in Hirschwang kann als eine gruppendynamische - Reaktion auf diese vielfältigen Herausforderungen, denen sich gegenwärtige Gesellschaften ausgesetzt sehen, gelesen werden. Ausgehend von vielfältigen Überlegungen wurde im Hirschwanger Staff ein Lernsetting entwickelt, das versucht diesen komplexen, gesellschaftlichen Anforderungen gerecht zu werden.

Beginnend bei medienhistorischen Überlegungen und Erklärungen von digitalem und medialem Wandel setzt sich dieser Artikel mit der These Auseinander welche Parallelen es zwischen prozess- und handlungsorientierten Prinzipien der Medienbildung und des gruppendynamischen Lernens gibt. Beide Themenkomplexe werden als Basis einer demokratierelevanten Auseinandersetzung diskutiert und finden Eingang in die Schilderungen bezüglich des Organisationslaboratoriums in Hirschwang.

\section{Veränderungsprozesse, Herausforderungen und die Notwendigkeit neuer Strategien}

Medialer Wandel und Digitalisierung verlangen von uns allen Umgangsformen und Strategien mit Technologien zu entwickeln. Diese Herausforderung in sich ist nie abgeschlossen. Kaum ist eine neue App oder Plattform antizipiert, sind wir mit einer neuen konfrontiert. Unser Medienkonsum ist nicht mehr nur Konsum, sondern oft auch Produktion. Der Alltag vieler Menschen ist von Mediennutzung und digitalen Tools in einer Selbstverständlichkeit durchdrungen, dass Trennlinien kaum mehr auszumachen sind. Gültigkeit hat dies für das Berufsleben genauso, wie für private Bereiche. Um mit diesen Anforderungen in tagtäglichen Situationen umgehen zu können, braucht es Steuerungs- und Medienkompetenzen, vor allem aber aktive Auseinandersetzung. Digitalisierung ermöglicht Vieles, transformiert aber auch umfassend und grundlegend: Virtuelle Zusammenarbeit, Zugriff und Umgang mit Daten und Informationen, Online-Shopping, digitale Vernetzung von Geräten oder Interaktionen mit KundInnen sind nur einige Beispiele dafür was sich alles ändert und noch ändern wird. Im Bereich ihrer Öffentlichkeit(en) sind Gesellschaften mit Phänomenen wie Fake News, Trollarmeen oder Verschwörungstheorien konfrontiert. Prozesse der Meinungsbildung, früher vorrangige Aufgabe von Massenmedien, funktionieren aufgrund sozialer Medien inzwischen gänzlich anders. Druck ausgesetzt sind dabei neben den Medienorganisationen vor allem auch politische Sphären und damit die Demokratien, die häufig noch keine adäquaten bzw. neuen Umgangsformen für diese digital-sozialen Phänomenen gefunden haben und darum ringen bestehende Strukturen mit Veränderungsprozessen und neuen Strategien in Einklang 
zu bringen. Gemeinsam ist den Veränderungen eine $\mathrm{Zu}-$ nahme an Komplexität, weitgehende Vernetzungen unterschiedlichster Bereiche und die Beschleunigung all dieser Prozesse.

Für ein besseres Verständnis dieser Herausforderungen hilft ein Blick in die Vergangenheit und eine Betrachtung des medialen Wandels und der Schritte, die historisch gesehen Einfluss genommen haben.

\section{Medienwandel aus (kommunikations)historischer Perspektive}

Die Menschheitsgeschichte lässt sich (auch) anhand ihrer Medienentwicklungen beschreiben. In engem Zusammenhand dazu stand, und steht, immer auch die Entwicklung der Demokratien: Der Buchdruck brachte eine Demokratisierung von festgehaltenem Wissen mit sich und ließ in weiterer Folge neben einer Verbreitung von Büchern auch die Formate Flugblätter, Zeitschriften und Zeitungen entstehen. Mit diesen Medien ${ }^{1}$ ging die Entwicklung von Journalismus, aber auch von Zensur einher. Erste massenmediale, und dabei auch demokratierelevante, Phänomene und Versuche der Limitierung resultierten aus diesem Entwicklungsschritt. Telegraph und Telefon führten zur medial vermittelten Kommunikation zwischen Personen. Die Verbreitung des Radios sorgte für die Entstehung einer massenmedialen Audiokultur und der Entwicklung von Propaganda und deren Strategien zur Einflussnahme auf gesellschaftliche Prozesse. Für die Zeit des Nationalsozialismus und 2. Weltkrieges ist diese mediale Evolution konstitutiv. Mit der Entstehung des Stumm- und später Tonfilms in der ersten Hälfte des 20. Jahrhunderts und dem Einzug von Fernsehgeräten in die Wohnzimmer der Menschen in der zweiten Hälfte, kam es zu einem schrittweisen Wandel der medialen Kultur hin zu einer stärker visuellen Ausrichtung. Die nun empfangbaren Bilder präg(t)en die Erinnerungen von Generationen. Vgl. dazu (Wilke 2003).

Gemeinsam ist all diesen Medien, und auch den weiteren dieser Epoche, eine Klarheit in Bezug auf die Positionierungen von Sender- und EmpfängerInnen. Die Rollen sind klar verteilt. Auf der einen Seite stehen die ProduzentInnen der Inhalte, die JournalistInnen, als aktiver Part der Medienproduktion. Dem gegenüber, meist gedacht als passive(re) Elemente, stehen die RezipientInnen, als jene an die sich mediale Inhalte richten. Lange Zeit wurde, sowohl im öf-

\footnotetext{
${ }^{1}$ Der Begriff Medium bzw. Medien wird an dieser Stelle des Artikels dem klassischen, kommunikationswissenschaftlichen Kanon entsprechend gefasst: Medien sind Mittel der Kommunikationsübertragung. Massenmedien sind Medien, die sich an eine große Anzahl an EmpfängerInnen richten. Vgl. dazu (Pross 1970, S. 129) oder (Saxer 1998, S. 54).
}

fentlichen, als auch wissenschaftlichen Diskurs, von einer Wirkmächtigkeit der Medien, deren Gatekeeperfunktionen und dem Festlegen von öffentlicher Agenda ausgegangen. Eine Durchlässigkeit zwischen den Positionen der Produktion und Rezeption war nicht gegeben oder vorgesehen. Die Medienlandschaft verfügte demnach über ausdifferenzierte Berufssparten, recht klare Strukturen und vor allem über diesbezügliche Eindeutigkeit.

Neben sozialen und ökonomischen Funktionen erfüllen Massenmedien eine wichtige Aufgabe als vierte Säule bzw. Gewalt im Staat. Durch ihre - idealer Weise - Unabhängigkeit garantieren sie Presse- und Meinungsfreiheit und nehmen ihre Informationsfunktion und Kontrollaufgaben gegenüber Machthabenden, wie PolitikerInnen oder WirtschaftsvertreterInnen, wahr. Wenn auch nicht alle, so pochen die meisten JournalistInnen auf die Freiheit der Presse und wehren sich gegenüber Einflussnahme aus politischen Sphären.

\section{Mediale Logik(en $)^{2}$ und deren Implikationen}

Jedes Medium verfügt über eine ihm eigene Logik: Bücher, wie z. B. Romane, werden linear vom Beginn bis zum Ende gelesen. Selbst wenn nur Auszüge eines Buches gelesen werden, bleibt es bei dieser Linearität und die jeweilige Stelle wird dieser Logik folgend rezipiert. Ähnlich verhält es sich beim Zeitungskonsum. Zwar unterscheiden sich die Strategien, wie eine Zeitung gelesen wird - so beginnen manche beim Sport- und andere beim Politikteil, das Lesen hat aber immer linearen Charakter. Bei Fernsehprogrammen kann zwar der Sender gewählt werden, nicht aber welches Programm ausgestrahlt wird. Die Programmierung des Senders obliegt diesem selbst, die NutzerInnen können sich in diesen Prozess kaum einbringen. Werbeunterbrechungen und sonstige Interventionen müssen diese während des Konsums akzeptieren. Ebenso verhält es sich beim Radio. Konsumiert werden kann, was angeboten wird, nicht aber ein selbst zusammengestelltes Programm. Die Haltung die RezipientInnen dieser Medien einnehmen, ist Konsum - diese sind KonsumentInnen von Inhalten, die andere schaffen. Eine treffende Bezeichnung für diese mediale Orientierung von KonsumentInnen traf Helene Katz mit der Bezeichnung des lean back oder lean backward. (Katz 2010, S. 3) Das dafür treffendste Beispiel ist der

\footnotetext{
${ }^{2}$ Dieses Wort im Plural widme ich Florian Klenk, dem Chefredakteur des Falters, der mir diesbezüglich in einer Diskussion auf Facebook widersprochen hat und meinte das Wort Logik gäbe es nur im Singular. Im diskutierten Fall der Gegenüberstellung unterschiedlicher Medien, bleibt aber nichts anderes als den Medien verschiedene Logiken zuzuordnen, da Ausrichtung, Wirkung und Nutzung sich jeweils logisch
} unterscheiden. 
Fernsehkonsum auf der Couch: Gemütlich zurückgelehnt, mit der Fernbedingung in der Hand, wird durch die verfügbaren Programme gezappt bis an passender Stelle Halt gemacht und das Programm konsumiert wird.

Der Erwerb von Medienkompetenzen und -bildung fokussierte in dieser Zeit vor allem auf die kritische Auseinandersetzung und das Wissen um Medienformate und deren Anforderungen. MedienrezipientInnen sollten um ihren Konsum und die dahinterliegenden Haltungen wissen und Inhalte analysieren bzw. kritisieren können. Zwar standen ebenso ökonomische und strukturelle Auseinandersetzungen am Plan, der Erwerb von Medienkompetenzen wurde aber mit dem Erreichen eines gewissen Levels als mehr oder weniger abgeschlossen betrachtet. Bis zur Entstehung des Internets und allen darauf folgenden Veränderungen war dies eine adäquate Herangehensweise, da sich die Medienlandschaft nur auf der Ebene ihrer Formate, nicht aber grundlegend änderte.

\section{Medialer Wandel - die Medienwelt in der Krise}

Bis in die 90er Jahre hinein blieben also die Verhältnisse fix, die Unterscheidungen in elektronische und Printmedien machte Sinn und Berufsbilder blieben konstant. Auch ihre gesellschaftliche Funktion und Wirkmächtigkeit behielten Medien bis zu diesem Zeitpunkt. Mitte der 90er Jahre begann ein Wandel von dem heute noch viele der AkteurInnen überrascht zu sein scheinen. Die teils heute noch als ,neue Medien“ - etwas absurd, da doch schon mehr als 20 Jahre alt - titulierten neu entstandenen Wege brachten die alte, mediale Ordnung durcheinander. Damalige Neuerungen wie Emails führten zu einer Beschleunigung von Kommunikation, ließen Kommunikationswege verflachen und machte Auseinandersetzung mit schriftlicher Kommunikation notwendig. Das WWW und seine Websites machte Informationen zugänglich und sorgte damit - ähnlich wie der Buchdruck Jahrhunderte zuvor, jedoch um einiges schneller - für eine Demokratisierung von Inhalten, jedoch aber auch für eine Verbreitung skurriler Inhalte. Neu war, dass nun alle Menschen zu MedienproduzentInnen werden konnten. Die Dualität von Produzent- und RezipientInnen verschwand zunehmend und mit weiteren Entwicklungen verblassten die Eindeutigkeiten. Ende der 00er Jahre verbreiteten sich soziale Medien, wie z. B. Facebook oder Twitter: Nun konnten sich alle mit Leichtigkeit einen Onlineauftritt zulegen und Teil der digital-sozialen Welt werden. Im Laufe der Jahre kamen unzählige neue Plattformen, Anwendungen oder Apps hinzu und ebenso viele verschwanden wieder im digitalen Nirwana. Digitale Prozesse und Kommunikationswege hielten in allen Lebensbereichen Einzug, kaum ein Gesellschaftsbereich konnte sich diesen Veränderungs- prozessen entziehen. Einen Ein- und Überblick über jeden Winkel der digitalen Welt kann heutzutage niemand mehr haben, so vielzählig und komplex sind die Angebote.

Das Nutzungsverhalten vieler, vor allem jüngerer, Menschen sieht heute anders aus: Während im schon geschilderten lean backward eine eher passive Konsumhaltung das Leitmotiv war, herrscht nun verstärkt eine Orientierung in Richtung lean forward. Medieninhalte werden selbstgesteuert ausgewählt und das Programm damit quasi selbst gemacht. Aktive NutzerInnen agieren auf Basis des Pull-Prinzips, das Push-Prinzip herkömmlicher Medien funktioniert bei diesen nur mehr bedingt - deren Angebote und Strategien greifen im Zeitalter der Digitalisierung nicht mehr:

„Overall, the locus of much cultural activity today is shifting from structure and tradition (push) to individual persons and their chosen networks that are composed of varying degrees of proximity and mediation (pull)." (Lull 2006, S. 45).

Medienmachende stellt dies vor ein Dilemma. Neue Angebote zu schaffen, zieht nach sich grundlegend in Abläufe und Strukturen einzugreifen. Eine Unterscheidung nach Medienformaten, wie Print oder Online, macht zunehmend weniger Sinn.

\section{Medien in der Krise - das digitale Jammertal}

Die einstige Ordnung des Mediensektors wurde und wird also durch diese Veränderungsprozesse auf den Kopf gestellt. Althergebrachte Verfahrensweisen funktionieren inzwischen nicht mehr. Überall steigt die Komplexität von Strukturen und Anforderungen und schafft damit Notwendigkeiten für neue Herangehensweisen und Strategien. Dass dies häufig versucht wird zu negieren oder zu umgehen, ist ein Phänomen dieser Überforderungen:

„Das Hereinbrechen der digitalen Ära überraschte und überforderte viele Medienbetriebe, deren Antworten und Lösungsstrategien sollten sich häufig als kontraproduktiv erweisen. Vor allem aber steht hinter dem Medienwandel auch und vor allem ein Wandel der Gesellschaft und der Öffentlichkeit." (Körber und Schaffar 2013, S. 8).

Die Organisationsstrukturen der Medienorganisationen konnten nicht - und können immer noch nicht - mit diesen Veränderungen mithalten. Sie blieben im Alten und Gewohnten während im Bereich der Produktion kein Stein am anderen blieb. Diese Krise stellt für Medien eine Überforderung dar und ist vielfach auch eine Krise des Managements, wie die Autorin, in Kooperation mit dem Journalisten Christian Körber, 2013 ausgeführt hat. Nicht nur sind die Medien und ihre Produkte in der Krise, auch ihre Organisationen stehen vor schwer zu bewältigenden Herausforderungen. Zwar wird um Antworten gerungen, Lösungen 
sind aber für viele dieser Unternehmen auch 2019 nur bedingt greifbar.

\section{Digitalisierung und Mediatisierung der Gesellschaft}

Auch politische und gesellschaftliche Sphären bzw. demokratische Prinzipien sind wegen des technologischen Wandels in der Krise. Das Phänomen der Fake News als eine dominante Strategie aktiver PolitikerInnen, wie z. B. des US Präsidenten Trump, sind zwar kein gänzlich neues Phänomen, stellen aber in ihrer Breite und öffentlichen Dominanz eine noch nie da gewesene Herausforderung dar. Heute dümpeln Verschwörungstheorien nicht mehr in dunklen Ecken von Hinterzimmern oder des Netzes, sondern sind gesellschaftlich breit präsent. Auch hier zeigt sich, dass übliche Strategien von NachrichtenjournalistInnen nicht greifen. Comedians und SatirikerInnen sind vielfach besser im Covern der Absurditäten und faktenlosen Behauptungen als Nachrichtensendungen und deren Anchors. Mit dem Überprüfen von Fakten und Aufzeigen von falschen Behauptungen sind diese immer einen Schritt hinter jenen, die diese aufstellen. Und selbst, wenn mit ihren eigenen Aussagen konfrontiert, sagen sie sich mit Leichtigkeit von jenen los. Was nicht sein soll, ist nicht, scheint hier als Prinzip zu funktionieren. Das auch deshalb, weil die Diversität des Medienkonsums NutzerInnen heute oft in Medienrealitäten hält aus denen diese nur schwer ausbrechen können. $\mathrm{Zu}$ wissen was richtig oder falsch ist, ist in einer digitalisierten Welt uneindeutig und fällt deshalb schwer.

Auch die wissenschaftliche Theoriebildung musste sich diesen Phänomenen stellen und neue Ansätze entwickeln: Dualitäten wie ProduzentInnen und RezipientInnen bzw. SenderInnen und EmpfängerInnen funktionieren, wie ausgeführt, schon lange nicht mehr. Richtungen sind in einem medialen Netzwerk mit unzähligen Knotenpunkten nicht mehr auszumachen. Eine medientheoretische Antwort darauf stellt das Konzept der Mediatisierung mit ihrem Fokus auf Wechselwirkungen dar:

„Mediatisierung ist hier ein Konzept, um die Wechselbeziehung zwischen medienkommunikativem und soziokulturellem Wandel kritisch zu analysieren. Hierbei sind mit dem Mediatisierungsbegriff quantitative wie auch qualitative Aspekte verbunden. In quantitativer Hinsicht fasst Mediatisierung die zunehmende zeitliche, räumliche und soziale Verbreitung von medienvermittelter Kommunikation. In qualitativer Hinsicht wird mit Mediatisierung der Stellenwert der Spezifika verschiedener Medien im und für den soziokulturellen Wandel gefasst." (Hepp 2014, S. 191).

Der Begriff und Ansatz der Mediatisierung hilft aus kommunikationswissenschaftlicher Sicht der Komplexität von Entwicklungen gerecht zu werden und einen neuen Rah- men zur Analyse zu setzen. Ähnlich wie die - und verwandt mit der - Praxeologie im Bereich der soziologischen Theorieentwicklung wird die wissenschaftliche Perspektive auf Situationen gelenkt. ${ }^{3}$ Der Blick der Forschenden wird mit dieser theoretischen Wendung in Richtung von beforschbaren Einheiten gelenkt. Anhand von Situationen können diese in den Blick genommen und deren Prinzipien, Bedingungen und Strukturen analysiert werden. Steht praxeologisch eine soziale Situation und deren inhärente Praktiken im Fokus, so werden mit Hilfe der Theorie der Mediatisierung mediale Situationen im Verhältnis zu soziokulturellen Veränderungen diskutiert.

\section{8 "Neue" Medien - neue Medienkompetenzen, eigentlich -bildung}

Komplexität und der Umgang mit dieser wird zu einer grundsätzlichen Herausforderung. Um Phänomenen wie Fake News zu begegnen, braucht es für alle Seiten neue Strategien und Auseinandersetzungsformen. Vor allem aber braucht es die Entwicklung grundlegender media literacy auf der Basis der vielfältigen medialen Prinzipien. Der englischsprachige Begriff fasst die Notwendigkeiten wesentlich treffender als der deutsche Begriff der Kompetenz. Kompetenz als Begrifflichkeit vermittelt eine Abgeschlossenheit: Ist die Kompetenz erworben, dann ist der Erwerb beendet. Der Prozess der Aktualisierung von Medienkompetenz ist aber ein nie abgeschlossener, der ein Leben lang betrieben werden muss.

Im medienpädagogischen Diskurs wurde Medienkompetenz deshalb durch den Begriff der Medienbildung ergänzt und erweitert. Vgl. dazu u. a. (Moser 2003). Hinter dieser begrifflichen Veränderung steht die Notwendigkeit auf das Prozesshafte und Veränderliche von medialen Kompetenzen zu verweisen. Die diesbezüglichen Fähigkeiten können nicht abschließend erworben werden, immer bleibt bedingt durch digitale bzw. mediale Innovationen und der Geschwindigkeit dieser - das Ende offen. Um medienkompetent zu sein und auch zu bleiben, muss man am Ball bleiben, sich stetig informieren, über neue Apps, Trends oder Plattformen Bescheid wissen und in Bewegung bleiben. Dies bedingt kein faktenbasiertes Wissen, sondern ein Wissen das auf dem Umgang mit Prozessen und Dynamiken basiert. Menschen müssen, um in diesem Gebiet Kompetenz aufzuweisen, wissen wie digitale Klippen umschifft werden können, müssen navigieren und fähig zur Selbststeuerung, vor allem aber fähig zum eigen- und selbstän-

\footnotetext{
${ }^{3}$ Stellvertretend für viele andere wird an dieser Stelle Andreas Reckwitz angeführt, der schon 2003 in seinem Artikel „Grundelemente einer Theorie der sozialen Praktiken.“ (Reckwitz 2003) die Notwendigkeit einer solchen Theoriebildung ausführte.
} 
digen Denken, sein. Da niemand vorhersagen kann welche Entwicklungen und Wendungen der technologische Wandel einschlagen wird, braucht es vielmehr die Fähigkeiten mit diesen Veränderungen umzugehen. Dies beinhaltet im Doing und aus Fehlern zu lernen, wie auch langfristig offen für Neues zu bleiben. Alles Anforderungen die viele Menschen, aber auch Organisationen und Gesellschaften vor komplexe Aufgaben stellen.

\section{Synchronisierung von Organisations- und Lernformen}

Der digitale Wandel bringt einen Anpassungsbedarf bezüglich organisationeller Strukturen mit sich. Im österreichischen Bildungssystem liegen didaktische Schwerpunkte im Unterricht auch heute noch bei Frontalvorträgen. Schulisches und universitäres Lehren und Lernen steht damit in einer Tradition der Wissensvermittlung. Die dem entsprechenden Organisationsformen von Bildungsinstitutionen sind klassische, funktionale Hierarchien.

Frontalunterricht als Format kann aber den Erwerb prozess- bzw. handlungsorientierter und selbstgesteuerter Kompetenzen kaum ermöglichen, da diese nicht vermittelt werden können. Nur veränderungsorientierte Lernszenarien mit Schwerpunkten, wie z.B. Projektunterricht oder spielerischen Settings, können einen passenden Rahmen bereitstellen, um Kompetenzen für Digitalisierungsprozesse zu erarbeiten. Um diese zu ermöglichen bräuchte es dementsprechende Organisationsstrukturen, die auf einen höheren Grad an Selbststeuerung ausgerichtet sind. Als These kann hier festgehalten werden: Erst wenn eine Synchronität zwischen Organisationsformen und Lernszenarien hergestellt wird, kann der Erwerb digitaler und medialer Kompetenzen im schulischen und universitären Rahmen gelingen.

Ähnliches gilt auch für Unternehmen: Diese sind gefordert mit dem digitalen Umbruch neue Arbeits- und Organisationsformen zu ermöglichen, da nicht nur Lern- sondern auch Arbeitsprozesse durch Digitalisierung grundlegend verändert werden. Behörden zeigen sich - langsam aber doch - bürgernäher und zugänglicher. Medienorganisationen bleiben mit Ihren RezipientInnen in Kontakt. Und selbst Baugenossenschaften beginnen zu realisieren, dass der Umgang mit ihren BewohnerInnen und deren Gemeinschaften aktiv adressiert werden muss. Schlichtwegs alle gesellschaftlichen Bereiche müssen sich den Veränderungen stellen, um nicht von diesen überrollt zu werden. Für diese Veränderungen braucht es veränderte Strukturen und auch Haltungen.

\section{Neue Organisationsformen und wo diese entstanden}

Ähnliche Phänomene, wenn auch aus anderer Perspektive, führten in der Branche der Informationstechnologien zu strukturellen Veränderungen: Der technologische Wandel ist in diesem Feld inhärenter Bestandteil des Organisationsalltags von IT-Unternehmen. Deren Aufgabenfelder, wie z.B. Programmierung und IT-Produktentwicklungen, setzen eine proaktive Auseinandersetzung mit neuen Technologien voraus.

Herkömmliche hierarchische Strukturen schaffen es nicht die notwendigen Möglichkeiten für innovative Entwicklungen bereitzustellen. Um innovativ produktiv zu sein, braucht es andere und neuartige Strukturen. Ideen, wie agile Programmierung oder z.B. auch Holacracy (Robertson 2015), entstanden und wurden enthusiastisch in dieser begeisterungsfähigen Branche implementiert. In ihrem Alltag offenbaren sich aber Lücken und Defizite bezüglich sozialer Strukturen und Dynamiken. Zwar können dynamische Organisationsansätze - Holacracy operiert mit einer ständigen Weiterentwicklung der Organisationsstrukturen in Form eines Govermentprozesses - einen passenden Rahmen zur Verfügung stellen, sehen aber keine Reglung des sozialen und zwischenmenschlichen Umgangs vor. Dass dies von den handelnden Personen aktiv geregelt werden muss, ist - wie in funktionalen Hierarchien auch Angelegenheit der Menschen in den Organisationen.

Organisationelle Veränderungen anzustoßen ohne (genügend) auf diese sozialen Prozesse und Entwicklungen zu achten, ist in keiner Organisationsform sinnvoll. Holacracy, als partizipative Organisationform mit demokratischen (Teil)Ansprüchen, kann ohne eine Entwicklung sinnvoller, kollektiver, sozialer Strukturen nur bedingt erfolgreich umgesetzt werden. Hier offenbart sich eine grundlegende Herausforderung für holakratische Organisationen. Das dominante Regelwerk suggeriert eine umfassende Regelung der organisatorischen Abläufe und das Erlernen dieser stellt für alle Beteiligten einen enormen Aufwand dar. Für die Ebene der Regeln und holakratischen Strukturierungen stimmt dies, die Verfassung von Holacracy regelt viel und umfangreich (HolacracyOne, LLC 2019). Etwas das Menschen, konfrontiert mit dieser Vielzahl an Regelungen und Vorgaben, oftmals aus den Augen verlieren: Soziale Prozesse können nur unzulänglich durch ein Regelwerk bestimmt werden. Ohne die Ebene eines Bewusstseins für die Gestaltung sozialer Räume und die Beziehungen zwischen den Organisationsmitgliedern fehlen wichtige Elemente für die organisationale Praxis. Auch wenn in Trainings und Literatur oftmals auf diesen Aspekt hingewiesen wird - vgl. dazu u. a. (Seuhs-Schoeller 2019) - überlagert der Eindruck des Geregelten häufig die Erkenntnis auch die Ebene der sozialen Prozesse anzusteuern und bearbeiten zu müssen. 
Demokratische Mitbestimmung und Partizipation in Organisationen umzusetzen, bedarf aber genau dieser Ebene, um sich langfristig und nachhaltig zu verankern. Wie auch in herkömmlichen Hierarchien müssen in Holakratien zwischenmenschliche Prozesse adressiert und bearbeitet werden. Die formellen Strukturen können eine Auseinandersetzung mit diesen nicht ersetzen. Wie in allen Organisationen ist dies auch in holakratischen ein Bereich in dem Bewusstseinsbildung für soziale Dynamiken und Prozesse betrieben werden muss und diese letztendlich auch bearbeitet werden müssen. Ähnliches trifft auch der Ebene der individuellen Skills zu. Die handelnden Personen müssen sich Prinzipien der Selbstorganisation erarbeiten, um im gesteckten Rahmen einer Holacracy tätig sein zu können. Diese Fähigkeiten zu erwerben, bedarf ebenso Praxis, wie auch Reflexion. Letztere ist im Arbeitsalltag nur schwierig zu erwerben, in dementsprechenden Lernsettings aber sehr wohl.

Womit diese Organisationen jedenfalls ausgezeichnet umgehen können, ist der technologische Wandel: Die grundsätzliche Haltung Innovationen $\mathrm{zu}$ integrieren und bezüglich dieser am Ball zu bleiben, wird von der Organisationform gestützt. Die Menschen in diesen Organisationen bleiben aufgrund dessen Veränderungen gegenüber neugierig - eine der notwendigen Konfigurationen um mit Effekten von Digitalisierung und medialem Wandel umgehen zu können.

\section{Zusammenhänge medialer und sozialer Dynamiken: Formate für den Erwerb von media literacy und Prozesskompetenzen}

Wenn also Komplexität zunimmt und die Richtung des fortschreitenden, medialen Wandels nicht vorhergesagt werden kann: Wo kann prozedurales Wissen erworben werden? Wo und in welchen Settings können Kompetenzen erworben werden, um medienkompetent und gesellschaftlich relevant agieren zu können?

Die Individuen einer Organisation zu schulen, ist nicht ausreichend - Medienbildung und -kompetenz lässt sich nicht vermitteln, sondern kann nur selbst erworben werden, weshalb Projektunterricht und Formate selbstgesteuerten Lernens bei MedienpädagogInnen schon lange hoch im Kurs stehen.

Und Erfahrungen aus organisationaler Praxis zeigen: Die Veränderungen von Organisationsstrukturen an sich sind nicht ausreichend. Ohne die Verankerung im Sozialen und der Ausbildung eines organisationsinternen Bewusstseins für kollektive Elemente und soziale Dynamiken bzw. Prozesse nützt die schönste, theoretisch partizipative Struktur nichts.

Alle skizzierten, durch den medialen Wandel hervorgerufenen, und notwendigen Veränderungen haben eines ge- meinsam: Sie sind alle auf der Ebene von handlungspraktischen und erfahrungsbasierten Prozessen angesiedelt. Sie können nicht ausschließlich faktenbasiert vermittelt, sondern müssen eigenständig erworben werden. So ist die Bedienung eines Emailclients vermittelbar, nicht aber der Umgang mit dem Medium Email, wie z.B. die Entwicklung von Strategien zum Verfassen von Emails. Diese müssen individuell erarbeitet und im Tun erlernt werden. Erst der Erwerb von Praxis in Kombination mit der Reflexion derselben macht die Herausbildung und stetige Weiterentwicklung medialer und digitaler Kompetenzen möglich.

Hier befindet sich die Brücke zwischen Medienbildung und gruppendynamischen Lernen: Beide Bereiche setzen voraus, dass nur im Tun und der Reflexion gelernt werden kann. Vgl. (Schattenhofer 2009) und (Süss et al. 2018, S. $97 \mathrm{ff}$.). Beide benötigen Settings, die in einem klaren Rahmen selbstgesteuertes Lernen ermöglichen. Die Implementierung und Vernetzung der Ansätze kann demokratiepolitisch wichtige Impulse setzen und TeilnehmerInnen Möglichkeiten eröffnen mit den Herausforderungen von Komplexität umzugehen, Strategien im Realen wie Digitalen $\mathrm{zu}$ entwickeln und diese auch im eigenen Tun zu verankern. Erfahrungsbasiertes Wissen und Handlungskompetenzen können so erlernt werden, um partizipative Organisationsformen auch langfristig und nachhaltig in ihrer Neuausrichtung zu unterstützen.

\section{Das Organisationslab: Praktisches Lernen von demokratisch relevanten Kompetenzen}

Ein praktisches Beispiel für den Ansatz des selbstgesteuerten Lernens ist das Organisationslaboratorium in Hirschwang: Dieses wurde von Barbara Kump und Joachim Schwendenwein begründet und ist eine Kooperation von FH Wien und TH Köln. Vier Jahre, bis zur Auflösung des Faches der kommunikativen Kompetenzen im Jahr 2018, war auch die Universität Wien daran beteiligt. Im aktuellen Staff des OLabs sind - in alphabetischer Reihenfolge: Babette Brinkmann, Barbara Kump, Matthias Lang, Andrea Schaffar und Joachim Schwendenwein. Organisationlaboratorien sind ein gruppendynamisches Lernsetting in dem reflexiv, prozess- und handlungsorientiert gelernt wird. In einem begleiteten Rahmen wird die Auseinandersetzung mit sozialen Dynamiken und deren Reflexion ermöglicht. Vgl. (Krainz 2008, S. 28) Das Hirschwanger Organisationslab findet jährlich 5-tägig in Klausurform statt. 2018 waren knapp 80 TeilnehmerInnen vor Ort.

2017 kam es zu einem Neudesign des Formates, um Veränderungsprozessen in Organisationen, wie auch dem digitalen Wandel gerecht(er) zu werden. Grundidee war drei unterschiedliche Organisationsansätze in drei Divisionen zu 
Abb. 1 Digitale Bausteine am Organisationslab Hirschwang, Darstellung: Andrea Schaffar

\section{Implementierung digitaler Bausteine}

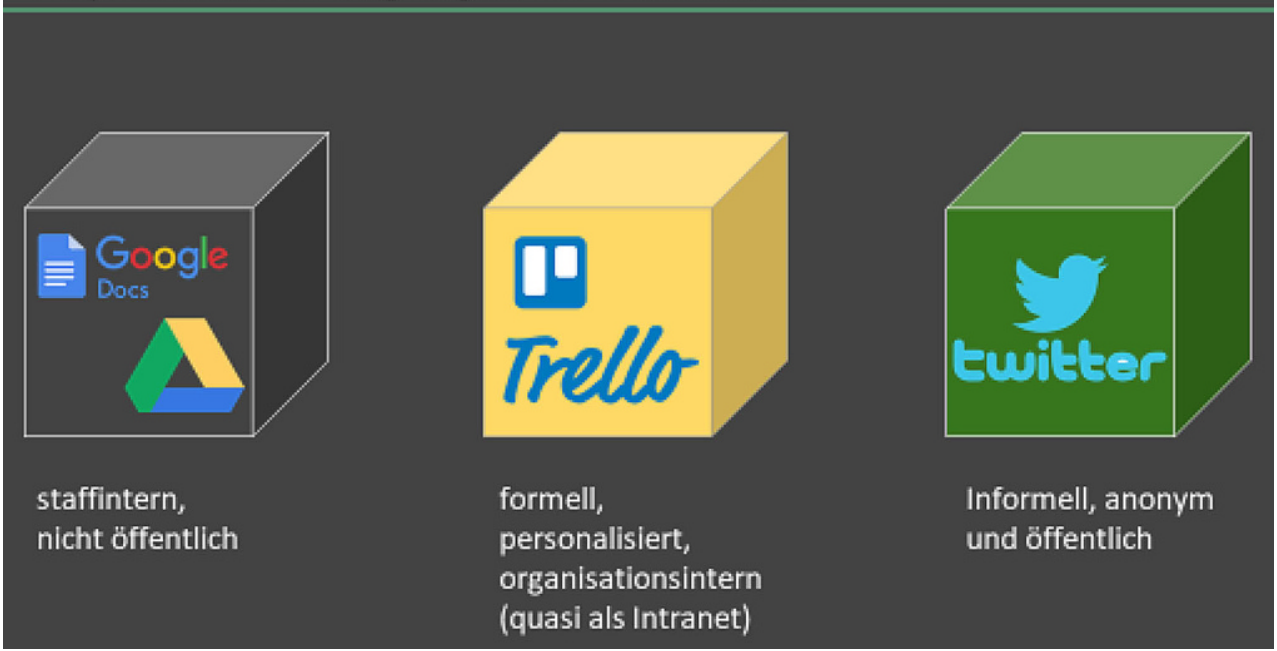

verankern und den Teilnehmenden so die Möglichkeit zu eröffnen diese zu erleben: Funktionale Hierarchie, Holacracy und Unternehmensdemokratie produzieren im Rahmen des Organisationslabs mit ihren unterschiedlichen Funktionsweisen bzgl. Partizipationsgrad gänzlich unterschiedliche Dynamiken und Prozesse. Eine Beschreibung der Überlegungen und des diesbezüglichen Ansatzes findet sich in der Publikation „Demokratie machen: Gruppendynamische Impulse“ im Beitrag „Selbststeuerung und demokratische Prozesse in der agilen Arbeitswelt" (Brinkmann 2018).

Zusätzlich zu dieser Grundkonstellation wurde als zusätzliche Ebene die Nutzung digitaler Kommunikationstools und -apps beschlossen. Ein Ausgangspunkt dafür war die unterschiedliche Technologienähe der jeweiligen Ansätze. Holacracy z. B. ist aufgrund der Entstehungsgeschichte und üblichen Durchführung sehr nah an digitale Tools gebunden, bei den beiden anderen Ansätzen ist dies nicht im gleichen Ausmaß gegeben. Schon in den Jahren vor dem Neudesign wurden digitale Elemente ins Setting integriert und ausprobiert. Ziel war es Phänomene der Einführung und Nutzung von digitalen Medien und Tools einer Reflexion zugänglich und dadurch ausgelöste Organisationsphänomene greifbar zu machen, was in den beiden bis jetzt stattgefundenen Durchgängen, als gelungen bezeichnet werden kann (Abb. 1):

1. Umgang mit Öffentlichkeit: Um die sonst kaum greifbare informelle Ebene aufzuschließen und eine Konfrontation mit den Prinzipien von Öffentlichkeit zu ermöglichen, wurde die Social Media Plattform Twitter als informeller und anonymer Kanals des OLabs gewählt. Die Accountdaten wurden zu Beginn bekannt gegeben und mit einem Hinweis auf Achtsamkeit und Öffentlichkeit zur Verfügung gestellt. Die Nutzung erfolgte schnell und intensiv.
Hauptsächlich wurden Memes und Kommentare veröffentlicht, die Stimmung im Rahmen des Labs wurde so gut greifbar. Ebenso wurde Twitter für Ankündigungen und Veröffentlichungen genutzt. Die Frage der Steuerung und Repräsentation stellte sich schnell und wurde insbesondere in den abschließenden, plenaren Reflexionen greifbar. Da bis auf den Anfangsimpuls keine weitere Steuerung des Staffs vorgenommen wurde, oblag die der Umgang der TeilnehmerInnen und ihrer Organisation selbst. Die Reflexion darüber, dass die digitale Kommunikation ebenso einer proaktiven Steuerung bedarf, wie organisationsinterne Kommunikation, kann als eines der Lernerlebnisse bezeichnet werden.

Begleitet wurde dieser Diskurs von einer Auseinandersetzung mit Datenschutz, der Verwendung von Photos von TeilnehmerInnen nur nach Zustimmung und damit einhergehend einer Reflexion von Persönlichkeitsrechten einerseits, der Gestaltung eines öffentlichen Auftritts andererseits.

2. Einführung digitaler Tools am Beispiel Trello, einer webbasierten Projektmanagementsoftware: In mehreren Jahren kam Trello als Element der Dokumentation zum Einsatz. Hier zeigte sich, dass die Art und Weise der Einführung einen Unterschied bezüglich der Akzeptanz des Tools ausmachte. Während in einem Jahr Trello von TeilnehmerInnen selbst eingeführt wurde und diesbezüglich hohe Akzeptanz in der Organisation herrschte, lief dies im darauffolgenden Jahr gänzlich anders. Als Vorgabe des Staffs, schon bei der Initiierung des OLabs und der Organisation genutzt, wurde Trello als Möglichkeit des Widerstandes genutzt - die TeilnehmerInnen erlebten dieses als Tool des Staffs und verhielten sich opposi- 
tionell. Im heurigen Jahr wurde auf eine Nutzung von Trello bei der Initiierung verzichtet, aber auf die Möglichkeit der Nutzung verwiesen und die Teilnehmenden nutzten dieses wiederum in einer selbstgesteuerten und akzeptierten Weise.

Auch wenn noch weitere Beobachtungen sinnvoll erscheinen, zeigt sich an diesen 3 organisationellen Situationen sehr klar, dass es auf die Art und Weise der Einführung und die handelnden Personen ankommt. Bei der Implementierung auf Freiwilligkeit zu setzen, erhöht die Akzeptanz enorm.

3. Digitales Protokoll via Google Docs: Eine digitale Neuerung betraf den Staff selbst. Da die Komplexität des Designs auf das Lab überging, wurde auch der Staff digitalisiert. Ein kooperatives, digitales Protokoll in das alle Staffmitglieder ihre Beobachtungen zeitgleich und live eintrugen, verringerten die Zeiten des Austausches und ermöglichten Einblicke in mehrere Orte gleichzeitig. Positiv daran ist den Verlauf des Labs jederzeit greif- und rekonstruierbar zu haben. Auch für künftige Forschungen sind diese seitenlangen Protokolle sicherlich aufschlussreich und nutzbar. Merkbar ist aber, dass die Nutzung eines solchen Tools auch negative Aspekte mit sich bringt. Für die Staffmitglieder erhöhen sich die Komplexität, der Aufwand und damit auch Stress. Das Protokoll bedarf einer kollektiven Aufmerksamkeit, die sich in manchen Situationen fordernd gestalten. Das Mitschreiben am Laptop oder Tablet zieht Aufmerksamkeit von TeilnehmerInnen auf sich und bedarf deshalb einer Auskommentierung zu Beginn des Labs. Für das staffinterne Lernen bzgl. digitaler Kommunikation eröffnet dieses aber weite Reflexionsfelder.

Am Beispiel des Hirschwanger Organisationslaboratoriums zeigt sich, dass das Schaffen von Bewusstsein für die Notwendigkeit kollektiver Prozesse im digitalen Zeitalter auch mit einer Auseinandersetzung von digitalen Formaten einhergeht. Das Erlernen von kollektiver Steuerung kann so auf die inzwischen in allen Organisationen vorhandenen digitalen und virtuellen Ebenen ausgeweitet werden. Teilnehmende werden so nicht nur in direkten sozialen Prozessen kompetenter, sondern auch in digital und medial vermittelten. Phänomene können so sowohl im sozialen als auch im digitalen Raum beobachtet, analysiert und reflektiert werden. Daraus resultiert eine höhere Befähigung in direkter als auch digitaler Interaktion zu navigieren, zu steuern und damit alle vorhandenen Ebenen zu adressieren. Durch die Implementierung eines digitalen Raumes im Lab öffnen sich andere Referenzsysteme und Phänomene wie Öffentlichkeit und Organisationsumwelt rücken stärker in das Blickfeld der Analyse.
Eines der größten Assets der gruppendynamischen Settings, das Erlernen und der Umgang mit Perspektivität und Unterschieden, kann so auch auf Digitales transformiert werden. Die Handlungspraxis in der im Lab entstehenden Organisation erweitert sich so von zwischenmenschlicher, formeller wie informeller Kommunikation, auch auf medial vermittelte. Die unterschiedlichen Prinzipien und Logiken werden sicht- und damit analysierbarer.

\section{Demokratische Relevanz}

Letztendlich sind es genau diese Phänomene und Dynamiken, die auch in Demokratien Relevanz haben. Die Digitalisierung bringt neue Anforderungen, aber auch Ansprüche mit sich. Die Interaktion zwischen politischen AkteurInnen und deren WählerInnen hat sich, ebenso wie die schon ausgeführten zwischen Medien und RezipientInnen, verändert. Von PolitikerInnen wird via Social Media Kanälen Kontakt und Reaktion eingefordert, um glaubwürdig zu bleiben. Meinungsbildung und politische Organisation - inner- und außerhalb politscher Parteien - findet vielfach auf digitalen Ebenen statt. Die Prinzipien digitaler Kommunikation sind jedoch andere als jene der massenmedialen oder direkten Interaktion. Fake News oder auch die einfache $\mathrm{Zu}$ gänglichkeit von Verschwörungstheorien und die Existenz medialer Parallelwelten stellen eine Bedrohung für demokratische Prinzipien dar. Dieser Komplexität zu begegnen, bedarf Lernsettings, die diese integrieren und Möglichkeiten eröffnen Strategien des individuellen, wie auch kollektiven, Umgangs zu entwickeln. Das Hirschwanger Organisationslaboratorium liefert dafür Beispiele: Der Umgang mit Öffentlichkeiten inner- und außerhalb der entstehenden Organisation rückte ebenso in den Mittelpunkt, wie Fragen von Datensicherheit und Privacy. Die unterschiedlichen Geschwindigkeiten der drei Organisationsformen wurden greif- und verhandelbar. Fragen von Repräsentation und Koordination mussten verhandelt und Reflexionsräume geschaffen, wie auch gehalten, werden. Zufriedenheit, zeigte sich, war zu unterschiedlichen Zeitpunkten gänzlich anders verteilt. Diese Ungleichverteilungen in Kombination mit komplexen Anforderungen bzgl. Medien- und (Lern)Produktmanagement eröffnete Lernfelder, die in die abschließende Reflexion der letzten zwei Tage einfloss.

Durch diese Verbindung von Organisationslernen, Medienbildung, der Auseinandersetzung mit sozialen Dynamiken und partizipativen Prinzipien in all ihrer Unterschiedlichkeit werden Teilnehmende bezüglich sozialer Prozesse, Öffentlichkeiten und Medieneffekten sensibilisiert. Demokratie lebt von genau dieser Auseinandersetzung, Reflexion, Kritikfähigkeit und einem konstruktivem Umgang miteinander indem gelernt wird wie mit Differenzen und Diversität zielführend umgegangen werden kann. 
In den Worten von Oskar Negt lässt sich das abschließend so fassen: „Demokratie ist die einzige politisch verfasste Gesellschaftsordnung, die gelernt werden muss - immer wieder, tagtäglich und bis ins hohe Alter hinein. Ich suche nach Antworten auf die Frage, warum Menschen unter bestimmten Bedingungen ihren politischen Verstand verlieren und andere politische Urteilskraft zeigen und praktizieren - unter Umständen sogar unter Einsatz ihres Lebens.“ (Negt 2016).

Funding Open access funding provided by University of Vienna.

Open Access Dieser Artikel wird unter der Creative Commons Namensnennung 4.0 International Lizenz (http://creativecommons.org/ licenses/by/4.0/deed.de) veröffentlicht, welche die Nutzung, Vervielfältigung, Bearbeitung, Verbreitung und Wiedergabe in jeglichem Medium und Format erlaubt, sofern Sie den/die ursprünglichen Autor(en) und die Quelle ordnungsgemäß nennen, einen Link zur Creative Commons Lizenz beifügen und angeben, ob Änderungen vorgenommen wurden.

\section{Literatur}

Brinkmann, B. (2018). Selbststeuerung und Demokratische Prozesse in der Agilen Arbeitswelt. In F. Stähler \& M. Stützle-Hebel (Hrsg.), Demokratie Machen: Gruppendynamische Impulse (S. 57-64). Heidelberg: Carl-Auer.

Hepp, A. (2014). Mediatisierung | Medialisierung. In J. Schröter (Hrsg.), Handbuch Medienwissenschaft (S. 191-196). Stuttgart, Weimar: Metzler.

Holacracyone, L. L.C. (2019). Holacracy. Self-management practice for organizations. https://www.holacracy.org/constitution. Zugegriffen: 10. Juni 2019

Katz, H. (2010). The media handbook: a complete guide to advertising media selection, planning, research, and buying. New York: Routledge.

Körber, C., \& Schaffar, A. (2013). Verschlafener Wandel. Medien und das digitale Jammertal. Medien \& Zeit, 28(2), 8-23.

Krainz, E. (2008). Gruppendynamik als Wissenschaft. In P. Heintel (Hrsg.), Betrifft: TEAM: Dynamische Prozesse in Gruppen (S. 7-28). Wiesbaden: VS.

Lull, J. (2006). The push and pull of global culture. In J. Curran \& D. Morley (Hrsg.), Media and cultural theory (S. 44-58). Oxon: New York.
Moser, H. (2003). Von der Medienkompetenz zur Medienbildung. Diskurstheoretische Überlegungen. Medienwissenschaft Schweiz, (2), 26-34.

Negt, O. (2016). Der Politische Mensch. Demokratie als Lebensform. Göttingen: Steidl.

Pross, H. (1970). Publizistik: Thesen Zu einem Grundcolloquium. Neuwied: Luchterhand.

Reckwitz, A. (2003). Grundelemente einer Theorie der sozialen Praktiken. Zeitschrift für Soziologie, 32(4), 283-301.

Robertson, B. (2015). Holacracy: the new management system for a rapidly changing world. New York: Henry Holt.

Saxer, U. (1998). Mediengesellschaft: Verständnisse und Mißverständnisse. In U. Sarcinelli (Hrsg.), Politikvermittlung und Demokratie in der Mediengesellschaft. Opladen: Westdeutscher Verlag.

Schattenhofer, K. (2009). Was ist eine Gruppe? Sichtweisen und Unterscheidungen. In C. Edding \& K. Schattenhofer (Hrsg.), Handbuch alles über Gruppen: Theorie, Anwendung, Praxis (S. 16-46). Weinheim Und Basel: Beltz.

Seuhs-Schoeller, C. (2019). Self-organization: what is personal and what is organizational? https://energized.org/en/language-ofspaces/. Zugegriffen: 11. Juni 2019.

Süss, D., Lampert, C., \& Trültzsch-Wijnen, C. (2018). Medienpädagogik: Ein Studienbuch zur Einführung. Wiesbaden: Springer VS.

Wilke, J. (2003). Kommunikations- und Mediengeschichte. In G. Bentele, H.-B. Brosius \& O. Jarren (Hrsg.), Öffentliche Kommunikation. Handbuch Kommunikations- und Medienwissenschaft (S. 151-168). Wiesbaden: VS.

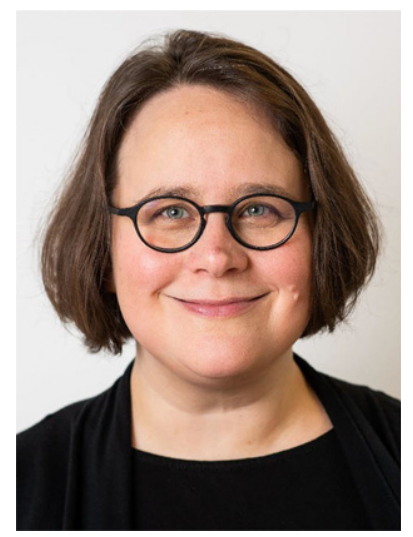

(c) Luiza Puiu
Mag.a Dr.in Andrea Schaffar Kommunikations- und Sozialwissenschafterin in Wien. Lehrt an der Universität Wien, TU Wien und TH Köln zu Digitalisierung, Medienbildung, Stadt- und Wohnbauforschung, Methoden der Sozialforschung, wie auch Gruppenund Organisationsdynamiken. Forschungstätigkeiten zu o.a. Themen. Mitglied des Hirschwanger OLab Staffs. Selbständig als Trainerin, Coach und Organisationsberaterin mit eigener Firma: Projektbüro XO KG, https://projektbuero.at/. Mitglied der ÖGGO, der Österreichischen Gesellschaft für Gruppendynamik und Organisationsberatung, Obfrau von FiZ Forschungsinstitut Zivilgesellschaft, https://fiz.ac.at/. 